\title{
Detection of minimal mucosal esophageal lesions in non-erosive gastroesophageal reflux disease using optical enhancement plus optical magnification $\square$
}

다(1)이우

\author{
Authors \\ Carlos Robles-Medranda, Manuel Valero, Miguel Soria-Alcívar, Miguel Puga-Tejada, Roberto Oleas, Jesenia Ospina, \\ Haydee Alvarado-Escobar, Jorge Baquerizo-Burgos, Hannah Pitanga-Lukashok
}

Institution

Gastroenterology and Endoscopy Division, Instituto

Ecuatoriano de Enfermedades Digestivas (IECED),

Guayaquil, Ecuador

submitted 19.11.2018

accepted after revision 16.3.2019

Bibliography

DOI https://doi.org/10.1055/a-0953-1844 |

Endoscopy International Open 2019; 07: E979-E986

(c) Georg Thieme Verlag KG Stuttgart · New York eISSN 2196-9736

Corresponding author

Carlos Robles-Medranda, MD, Head of the Endoscopy

Division, Instituto Ecuatoriano de Enfermedades Digestivas

- IECED, Av. Abel Romeo Castillo y Av. Juan Tanca Marengo,

Torre Vitalis, Mezzanine 3, Guayaquil, Ecuador

Fax: +59342109180

carlosoakm@yahoo.es

\section{ABSTRACT}

Background and study aims The aim of this study was to evaluate the diagnostic yield of endoscopy using optical enhancement (OE system) with optical magnification to predict reflux in non-erosive reflux disease (NERD) patients.

Patients and methods A prospective, non-randomized, single-blind study was performed from September 2015 to January 2016. Participants suffered from gastroesophageal reflux disease (GERD) symptoms and were assigned to the NERD group or the non-reflux disease control group based on endoscopic findings and a 24-hour $\mathrm{pH}$-impedance-monitoring test. Endoscopy using the OE system with optical magnification was performed in all patients to detect minimal mucosal esophageal lesions (MMEL), specifically abnormalities in the numbers, dilation, and tortuosity of intrapapillary capillary loops (IPCLs). Biopsies were obtained from each esophageal segment, and diagnoses from images were compared to diagnoses of reflux and inflammation using 24-hour $\mathrm{pH}$-impedance monitoring and histology, respectively.

Results Fifty-seven patients were included (36 in the NERD group, 21 in the control group). IPCLs were observed in $94.4 \%$ of cases in the NERD group and $38 \%$ of cases in the control group $(P<0.05)$. There were significant differences in IPCL abnormalities between groups that were associated with histologically identified inflammation. The sensitivity, specificity, positive predictive value, negative predictive value, and accuracy were $94.4 \%, 61.9 \%, 80.9 \%, 86.6 \%$, and $82.4 \%$, respectively. The 24 -hour $\mathrm{pH}$-impedance-monitoring test was used as the gold standard. The Kappa interobserver and intraobserver values were 0.85 and 0.90 , respectively.

Conclusion The OE system with optical magnification can detect MMEL and predict NERD with high sensitivity, accuracy, and interobserver and intraobserver agreement. Presence of IPCLs highly correlates with histologically identified inflammation.

Clinical.Trials.gov

NCT02575287

TRIAL REGISTRATION: Prospective, controlled, non-randomized, single-blind study NCT02575287 at clinicaltrials. gov

\section{Introduction}

Gastroesophageal reflux disease (GERD) affects up to $20 \%$ of the population in Western Europe and North America [1]. GERD's typical symptoms, such as heartburn and regurgitation once per week, affect patients' quality of life [2,3]. The disease may be diagnosed by symptoms through patient history or questionnaires, a positive response to proton-pump inhibitor (PPI), or establishing presence of pathologic reflux on a 24- 
hour $\mathrm{pH}$-impedance monitoring test; however, these methods have limited sensitivity and specificity [4].

Esophagogastroduodenoscopy (EGD) with white light endoscopy (WLE) is a standard technique for evaluating the esophagus in patients with GERD to detect GERD complications, such as esophageal erosion. However, up to $60 \%$ of patients with GERD who present with symptoms such as heartburn or acid reflux have normal endoscopic findings with WLE and are classified as non-erosive gastroesophageal reflux disease (NERD) cases, requiring further studies to establish a final diagnosis [5].

Previous studies using virtual chromoendoscopy with I-SCAN with high-definition technology have shown that this technique improves diagnosis of erosive reflux disease by $30 \%$, allowing detection of small inflammatory changes (short erosions or short Barrett's esophagus) that were not previously detected by standard endoscopy $[6,7]$. Nevertheless, I-SCAN can detect minimal mucosal esophageal lesions (MMEL). However, due to its low sensitivity, this technique should not be used to detect these lesions [8].

Recently, an image-enhanced endoscopic technology using pre-processor band-limited light called the Optical Enhancement system (OE system was developed by HOYA Co. (Tokyo, Japan) and is now equipped with endoscopic video equipment. The OE system combines digital signal processing with optical filters that limit the spectral characteristics of the illumination light, thus improving mucosal visualization. In addition, new scopes, called MagniView have been developed with the ability to combine high-definition imaging with optical magnification.

In the normal esophageal mucosa, submucosal vessels that pierce the muscle layer are connected to the arborescent vascular network. Intrapapillary capillaries arise from the fourth branch of the arborescent vessels into the epithelial papillae and form loops (IPCL). It has previously been reported that patients with GERD have an increased number of vascular lesions (dilation and tortuosity of the IPCLs), a greater number of microerosions and increased vascularity at the squamocolumnar junction relative to the control group [9].

The aim of this study was to evaluate patients with NERD using the new $\mathrm{OE}$ system and optical magnification to determine its diagnostic yield for detection of MMEL. The latter was of particular interest because presence of MMEL can be considered an early sign of GERD in NERD patients and is not detectable through WLE or I-SCAN technology. In addition, we sought to compare MMEL images to 24-hour $\mathrm{pH}$-impedance monitoring test and biopsy results to determine the sensitivity and specificity of the $\mathrm{OE}$ system in predicting inflammation.

\section{Patients and methods}

\section{Study design}

This was a prospective, controlled, non-randomized, singleblind study performed at the Instituto Ecuatoriano de Enfermedades Digestivas (IECED), Academic Tertiary Center, Ecuador, between September 2015 and January 2016. The study protocol and consent form were approved by the Institutional Review Board and registered at ClinicalTrials.gov (ID: NCT02575287).
The study was conducted according to the Declaration of Helsinki. Patients provided informed consent and answered a questionnaire to provide biometric data (sex, age, weight [kg], height $[\mathrm{m}]$, body mass index $\left[\mathrm{kg} / \mathrm{m}^{2}\right]$ ), and information about chronic diseases, medications and main reflux symptoms.

\section{Population selection and inclusion and exclusion criteria}

NERD group and control group selection

Patients were recruited from the gastroenterology unit of the IECED. Patient selection (including the control group) was performed after the following analysis ( $>$ Fig. $\mathbf{1}$ ). All patients with troublesome typical reflux symptoms (heartburn and regurgitation) for more than 6 months and with more than eight points on the Spanish validated version of the GerdQ questionnaire by Zavala-González et al ( Table 1) underwent EGD [10]. Before endoscopic examination, antisecretory therapy using PPIs was discontinued for 3 weeks.

We then performed a conventional complete endoscopic procedure that reached the Z-line of the esophagus, and evaluated the entire esophagus (upper, middle and lower segments) initially using high-definition WLE, followed by digital chromoendoscopy (I-SCAN Pentax, Tokyo, Japan) with three different image algorithms, including l-scans 1, 2 and 3 (tone and enhancement filters), that accurately detects erosive reflux, short Barret's esophagus and minimal change esophagitis (MCE) [7,11]. Presence of reflux lesions, such as ulcerative esophagitis, esophageal strictures, Barrett's esophagus or any erosive sign according to the Los Angeles classification (grade A to D) as well as MCE, was considered sufficient to classify patients as having erosive GERD, and they patients were excluded from the protocol [12]. On the other hand, if the esophagus was normal and no abnormalities were observed, a 24-hour $\mathrm{pH}$-impedance monitoring test (VersaFlex Z, Given Imaging, Yokneam Illit, Israel) was performed as the gold standard method to diagnose NERD [13]. Details of the technical background of the $\mathrm{pH}$-impedance procedure have been described previously [13]. GERD diagnosis was considered when there were more than 73 reflux episodes in 24 hours or when there was an abnormal acid exposure time (AET) with $\mathrm{pH}<4$ measured more than $4.2 \%$ of the time over 24 hours [14]. After this period, the investigators were able to discriminate between patients with NERD (patients with reflux symptoms, no lesions on EGD and with a positive 24-hour $\mathrm{pH}$-impedance monitoring test) and patients with functional heartburn (patients with reflux symptoms, no lesions on EGD, and a negative 24-hour $\mathrm{pH}$-impedance monitoring test). Two groups were selected: the NERD group, and the control group. The control group included patients with functional heartburn who agreed to be part of this study.

Included patients (NERD group and control group) were older than 18 years of age, had a history of GERD symptoms with more than eight points on the GERD questionnaire, and agreed to participate in this study. Patients with any type of esophagitis (actinic, caustic or eosinophilic), achalasia, esophageal varices, esophageal cancer, usage of PPIs or non-steroidal anti-inflammatory drugs (NSAIDs) for at least 3 weeks before the EGD, severe uncontrolled coagulopathy, gastric lesions (ulcer, 
Reflux symptoms + GERD Questionnaire (n: 141)

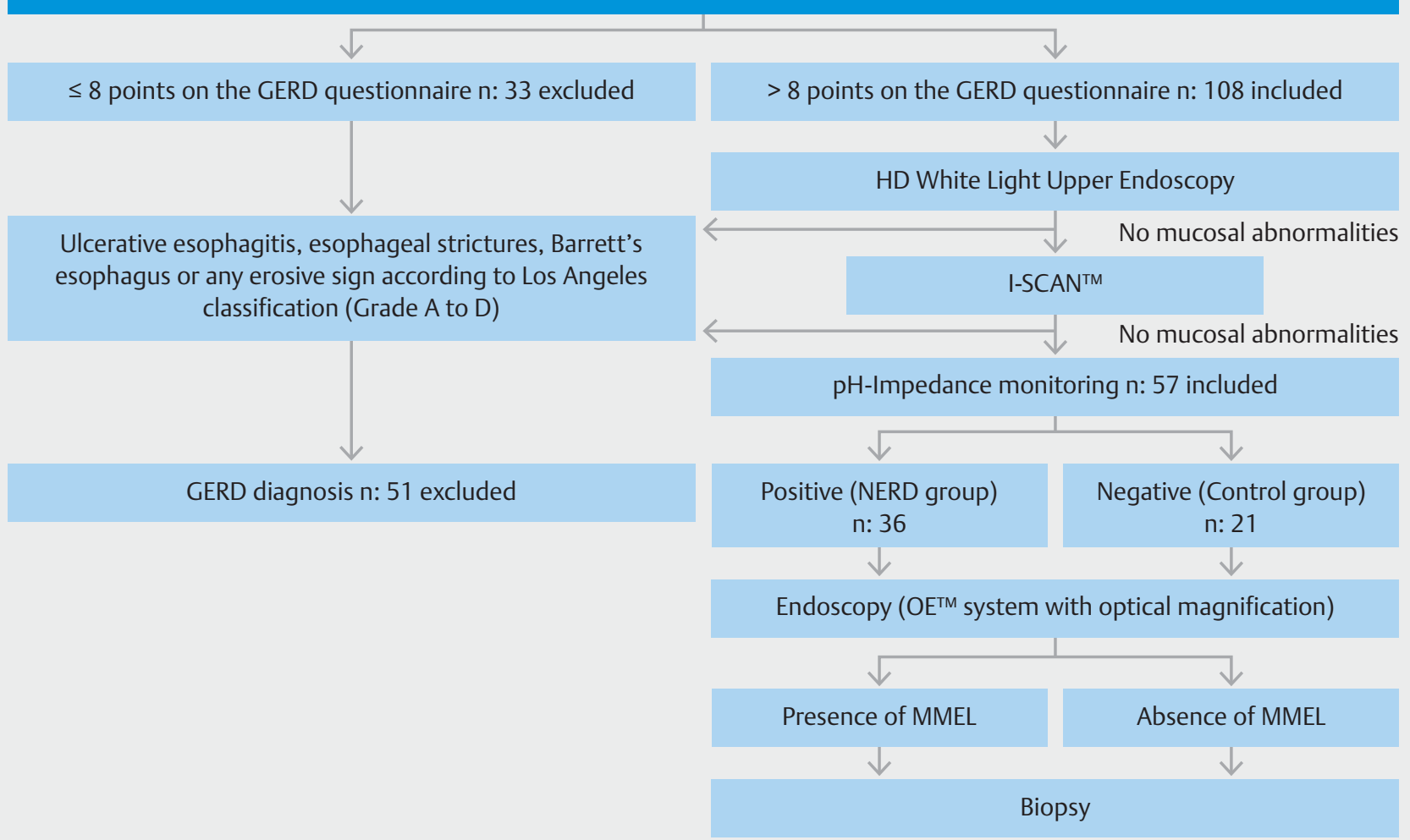

Fig. 1 Study flow diagram.

polyp, cancer), severe gastroparesis, and a history of esophageal/gastric surgery or pregnancy were also excluded.

\section{Endoscopic technique}

All patients included in the protocol (the NERD group and the control group) were evaluated by EGD using the OE system (EPK-i7010 processor) and MagniViewscopes (EG-2990Zi) (Pentax Medical, Hoya Corp., Japan). This technique involved use of a distal rubber hood (OE-A58) (Pentax Medical, Hoya Corp., Japan) at the tip of the scope. After reaching the Z-line, the four quadrants of the three esophageal segments (upper, middle and lower) were evaluated. First, the cup was allowed to contact the esophageal mucosa and water was ejected from it. Optical magnification was implemented using a button on the MagniView scope. Then, the OE system was activated using mode 1 (described below). All of the observed abnormalities were photographically recorded (with video and pictures) for further validation. Finally, biopsies were completed in both groups (one targeted biopsy from any lesion detected with $\mathrm{OE}$ system and optical magnification). Endoscopies were performed by three endoscopists (C.R-M, M.V, M.S-A) who were blind to group selection and were trained on the $\mathrm{OE}$ system with optical magnification.

\section{Optical Enhancement system (OE system) and Magniview scopes}

OE system

This technology combines digital signal processing with optical filters that limit spectral characteristics of the illuminated light. Earlier I-SCAN technology uses white light alone as an illumination source. Subsequent digital post-processing of the light's reflection creates images yielding the virtual chromoendoscopic image. The basic goal of $\mathrm{OE}$ is to overcome the darkness of narrow band imaging (NBI), which makes it more challenging to perform useful wide-range observations in the full extent of the gastrointestinal lumen. OE optical filters achieve higher overall transmittance by connecting the peaks of the hemoglobin absorption spectrum $(415 \mathrm{~nm}, 540 \mathrm{~nm}$, and $570 \mathrm{~nm})$, thereby creating a continuous wavelength spectrum. There are two modes that can be used with different OE filters. For the purpose of this study, Mode 1 was used exclusively given its ability to improve microvessel visualization using a sufficient amount of light.

\section{Magniview scopes}

This scope technology combines high-definition scopes with optical magnification. This technology magnifies images up to 136 times, leading to a more detailed image than standard scopes without optical zoom. This feature subsequently allows better evaluation of the superficial vascular aspects of the mu- 
- Table 1 GERD-Q questionnaire.

\begin{tabular}{|c|c|c|c|c|}
\hline \multirow[t]{2}{*}{ Questions } & \multicolumn{4}{|c|}{ Frequency score for symptoms } \\
\hline & $\mathbf{0}$ day & 1 day & $2-3$ days & 4-7 days \\
\hline How often did you have a burning feeling behind your breastbone (heartburn)? & 0 & 1 & 2 & 3 \\
\hline $\begin{array}{l}\text { How often did you have stomach contents (liquido } r \text { food) mooving upwards to your throat or } \\
\text { mouth (regurgitation)? }\end{array}$ & 0 & 1 & 2 & 3 \\
\hline How often did you have pain in the center of the upper stomach? & 3 & 2 & 1 & 0 \\
\hline How often did you have nausea? & 3 & 2 & 1 & 0 \\
\hline $\begin{array}{l}\text { How often did you have difficulty getting a Good night's sleep because of your heartburn and/ } \\
\text { or regurgitation? }\end{array}$ & 0 & 1 & 2 & 3 \\
\hline $\begin{array}{l}\text { How often did you take additional medication for your heartburn and/or regurgitation, other } \\
\text { than what the physician told you to take (such as Tums, Rolaids and Maalox? }\end{array}$ & 0 & 1 & 2 & 3 \\
\hline \multicolumn{5}{|l|}{ GERD-Q Spanish Version } \\
\hline Preguntas & Nunca & 1 día & 2 - 3 días & 4-7 días \\
\hline ¿Con qué frecuencia ha tenido sensación de quemadura detrás del esternón (ardor)? & 0 & 1 & 2 & 3 \\
\hline $\begin{array}{l}\text { ¿Con qué frecuencia ha notado que el contenido del estómago (líquido o alimento) le subía a } \\
\text { la garganta o a la boca (regurgitación)? }\end{array}$ & 0 & 1 & 2 & 3 \\
\hline ¿Con qué frecuencia ha sentido dolor en la boca del estómago? & 3 & 2 & 1 & 0 \\
\hline ¿Con qué frecuencia ha tenido náuseas? & 3 & 2 & 1 & 0 \\
\hline $\begin{array}{l}\text { ¿Con qué frecuencia tuvo problemas para dormir bien por la noche debido a ardor o } \\
\text { regurgitación? }\end{array}$ & 0 & 1 & 2 & 3 \\
\hline $\begin{array}{l}\text { ¿Con qué frecuencia tomó otros medicamentos para el ardor y/o la regurgitación aparte de } \\
\text { los que recetó su médico? (gel, sal de uvas u otro antiácido) }\end{array}$ & 0 & 1 & 2 & 3 \\
\hline
\end{tabular}

cosa, identifying early suggestive signs of inflammation or lesions not previously noted with conventional endoscopy.

\section{Minimal mucosal esophageal lesions}

All imaged esophageal segments (upper, middle and lower segments) were analyzed. Endoscopic images collected using $\mathrm{OE}$ with optical magnification were considered positive for MMEL if IPCLs (increase in number, dilatation, and/or tortuosity) were present ( $\mathbf{F i g . 2}$ ) [9].

For practical reasons and to reduce the time needed for the analysis, each fully magnified image was divided into four quadrants instead of counting IPCLs by field for evaluation of IPCL numbers. The IPCLs in each quadrant were manually counted, and the increase in number was determined when at least one quadrant had more than $30 \mathrm{IPCLs}$, as reported in a previous study [9]. A pilot analysis was performed with $10 \mathrm{pa}-$ tients from the NERD group and 10 from the control group, and a significant difference in the number of IPCLs was noted between groups. Dilatation of the IPCLs was recognized as an increase in twice the diameter of individual IPCLs, and IPCLs were considered dilated when they could be clearly observed at full magnification (136x magnification through the MagniView scope) $[9,15]$. Tortuosity was visually defined by presence of corkscrewing or by the twisted appearance of individual IPCLs ( $\triangleright$ Fig. 2) $[9,15]$.OE-derived images were compared with the results of the 24-hour $\mathrm{pH}$-impedance monitoring test and histological analysis of the biopsies to determine the sensitivity and specificity by which reflux and inflammation could be detected using $\mathrm{OE}$, respectively.

\section{Histologic inflammation criteria}

For biopsy analysis, tissues were considered inflamed due to reflux if neutrophil and eosinophil infiltration, papillary elongation, basal zone thickening (hyperplasia) and/or dilation of the intercellular spaces (DIS) was detected $[16,17]$.

\section{Interobserver and intraobserver agreement analysis}

A dataset containing 60 photographs of the three esophageal segments was presented to three blinded endoscopists who were asked to classify the photographs as negative or positive IPCLs (increases in IPCL number, dilatation and tortuosity) at three time points each 1 week apart. Each time, the same photographs were shown to the endoscopists but in a different order. The three endoscopists were trained to evaluate the three IPCL parameters. Interobserver agreement was measured by comparing the results of analysis of the photographs by each 


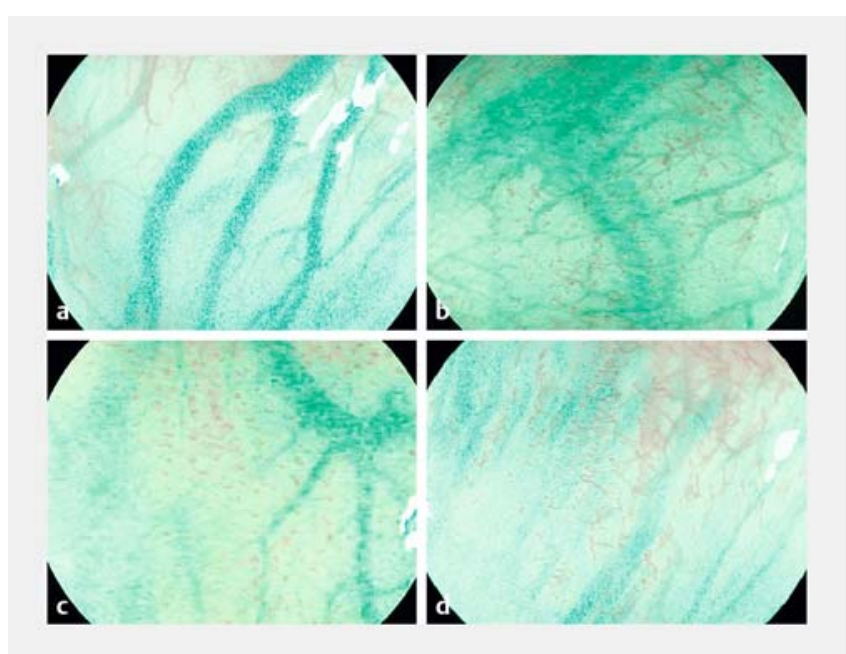

- Fig.2 An OE with an optical magnification endoscopy image showing a normal intra-papillary capillary loops (IPCLs); b tortuous IPCLs; $\boldsymbol{c}$ increased number of IPCLs; $\mathbf{d}$ dilated IPCLs from multiple NERD patients.

endoscopist (C.R-M., M.V., M.S.A.). Intraobserver agreement was measured based on a comparison of assessment of the photographs by the same three endoscopists at each time point.

\section{Statistical analysis}

The sample size needed to perform robust intra-rater and interrater analyses was calculated using Cohen's kappa. The required sample size was estimated by considering a kappa value under the null hypothesis of $50 \%$, alpha error (type I) of $5 \%$ and beta error (type II) of $20 \%$, which corresponded to a statistical power of $80 \%$. We considered the proportion of positive diagnoses per endoscopist and the previously known kappa value from flexible spectral imaging color enhancement feasibility for NERD [18]. NERD group and control group patients' baseline characteristics were compared using Student's $t$-test, Welch's $t$-test or the Mann-Whitney $U$ test for continuous variables and Pearson's chi-square test or Fisher's exact test for categorical variables. Continuous variables are expressed as the mean (standard deviation) or median (interquartile range) according to their statistical distribution. Categorical variables are expressed as percentages.

Visual OE image assessments were compared with results from the gold-standard 24-hour $\mathrm{pH}$-impedance monitoring tests and the biopsies to determine overall accuracy of the $\mathrm{OE}$ analysis. Diagnostic efficacy using OE was measured through sensitivity and specificity predictive values, and accuracy to predict reflux and inflammation at the $95 \%$ confidence interval $(95 \% \mathrm{Cl})$. To examine interobserver and intraobserver agreement, kappa values were calculated [16]. Kappa coefficients below 0.4 indicate "poor agreement," values between 0.4 and 0.8 represent "moderate to good agreement," and values greater than 0.8 indicate "excellent agreement".
$P<0.05$ was considered statistically significant. Data were assessed by an IECED institutional biostatistician. Statistical analysis was performed using R v3.4.3 (R Foundation for Statistical Computing; Vienna, Austria).

\section{Results}

\section{Patient baseline characteristics}

The estimated sample size required for robust interobserver and intraobserver analysis was calculated to be 46 patients, and 57 patients were finally included in the protocol, corresponding to a statistical power of $88.5 \%$. Of these, 36 patients $(63.1 \%)$ were in the NERD group and 21 patients $(36.8 \%)$ were in the control group ( $\triangleright$ Fig. 1$)$. There were no significant differences between these groups in terms of age, sex, main symptoms, or GERD questionnaire scores. Mean age of all patients was 48 years (14.1). Forty-six of 57 patients (81\%) were female, and the main symptom was regurgitation in 27 of 57 cases $(47.4 \%)$. The median GERD questionnaire score was 13 (interquartile range $8-18$ ) ( $\triangleright$ Table 2 ). Among groups, there were not any macroscopic lesions detected with WLE.

\section{NERD patients have higher numbers of MMEL than control patients}

There was a significant difference in the number of patients with MMEL detected with the OE system with optical magnification between the NERD group and control group.IPCLs were observed in 34 of 36 cases (94.4\%) in the NERD group and 8 of 21 cases $(38 \%)$ in the control group $(P<0.001)$ ( $>$ Fig. 2 and - Video 1).

A positive EGD was characterized by presence of IPCLs in at least one esophageal segment. A positive EGD was observed in 42 patients (34 in the NERD group and 8 in the control group, $P<0.001)$, and most patients with a positive EGD $(28 / 42$ [66.6\%]) had inflammation according to histopathological examination of biopsied esophageal tissue. These findings sug-

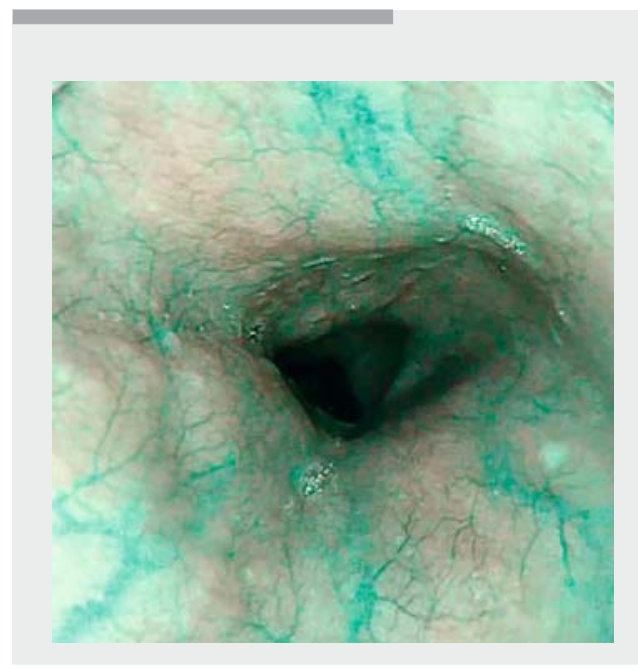

Video 1 Minimal mucosal esophageal lesions detected during OE with optical magnification endoscopy. Presence of tortuos and increased numbers of IPCLs. 
- Table 2 Baseline characteristics of the enrolled patients.

\begin{tabular}{|l|l|l|l|}
\hline Patient characteristics & $\begin{array}{l}\text { Total } \\
(\mathbf{n = 5 7 )}\end{array}$ & $\begin{array}{l}\text { NERD group } \\
\text { (n=36) }\end{array}$ & $\begin{array}{l}\text { Control group } \\
(\mathbf{n = 2 1 )}\end{array}$ \\
\hline Gender (Female: $\mathrm{n}, \%)$ & $46(80.7)$ & $29(80.6)$ & $17(81.0)$ \\
\hline Age (years) mean (SD) & $48.18(14.1)$ & $48.11(15.3)$ & $48.29(12.1)$ \\
\hline Symptoms, $\mathrm{n}$ (\%) & & & 0.971 \\
\hline - Heartburn & $8(14.04)$ & $5(13.9)$ & $3(14.3)$ \\
\hline - Regurgitation & $27(47.4)$ & $18(50.0)$ & $9(42.9)$ \\
\hline - Both & $22(38.6)$ & $13(36.1)$ & 9.969 \\
\hline GERD Questionnaire median (interquartile range) & $13(8-18)$ & $13(9-18)$ & $12(8-16)$ \\
\hline GERD, gastroesophageal reflux disease; SD, standard deviation. & & & 0.603 \\
\hline
\end{tabular}

- Table 3 Intrapapillary capillary loop characteristics in NERD group and control group patients.

\begin{tabular}{|c|c|c|c|c|c|c|c|}
\hline \multirow[b]{2}{*}{ IPCLs } & \multirow[b]{2}{*}{ EGD } & \multicolumn{3}{|c|}{ 24-hour pH-impedance monitoring test } & \multicolumn{3}{|l|}{ Biopsy } \\
\hline & & $\begin{array}{l}\text { Positive } \\
\text { (NERD group) }\end{array}$ & $\begin{array}{l}\text { Negative } \\
\text { (Control group) }\end{array}$ & $P$ value & Positive $^{1}$ & Negative & $P$ value \\
\hline Increase number, n (\%) & $31 / 42(73.8 \%)$ & $25(80.6 \%)$ & $6(19.4 \%)$ & $0.003^{1}$ & $20(64.5 \%)$ & $11(35.5 \%)$ & $0.025^{1}$ \\
\hline Dilatation, n (\%) & $33 / 42(78.5 \%)$ & $27(81.8 \%)$ & $6(18.2 \%)$ & $<0.001^{1}$ & $22(66.6 \%)$ & $11(33.4 \%)$ & $0.005^{1}$ \\
\hline Tortuosity, n (\%) & $24 / 42(57.1 \%)$ & $18(75 \%)$ & $6(25 \%)$ & $0.114^{1}$ & $15(62.5 \%)$ & $9(37.5 \%)$ & $0.134^{1}$ \\
\hline
\end{tabular}

gest that presence of IPCLs could be considered an indicator of reflux and inflammation in patients with NERD. Most patients with IPCLs had more than one esophageal segment with lesions; however, after analysis of the IPCL distribution by segment, it was found that the lower third of the esophagus was the most affected $(P<0.001)$.

\section{NERD patients have greater IPCL numbers and dilatation compared with control patients}

- Table 3 shows data for IPCL number, dilation, and tortuosity between groups. Sub-analysis revealed that IPCL dilatation was the most commonly observed lesion in patients with a positive UE (33/42 [78.5\%]). There was a significant difference in dilatation $(P<0.001)$ and numbers of IPCLs $(P=0.003)$, but not tortuosity $(P=0.114)$ between the NERD group and control group. Dilatation and increased numbers of IPCLs were more common among patients with biopsies with histopathologically detectable inflammation.

\section{OE system and Magniview scopes show high sensitivity, specificity and accuracy in predicting reflux and inflammation}

Overall calculated accuracy parameters are presented in $>\mathbf{T a}$ ble 4. Results from the visual analysis of the images of the esophageal mucosa obtained with the OE system and Magniview scopes were compared with the 24-hour $\mathrm{pH}$-impedance monitoring test to determine the ability of the OE system technology to predict reflux. Sensitivity, specificity, PPV, NPV, and accuracy were $94.4 \%, 61.9 \%, 80.9 \%, 86.6 \%$, and $82.4 \%$, respectively, and the use of the EGD in the lower third of the esophagus was associated with the highest sensitivity (88.9\%) and accuracy (87.7\%). Similarly, the OE system ${ }^{\mathrm{TM}}$ results were compared with targeted biopsy histopathology findings to determine the ability of the $\mathrm{OE}$ system ${ }^{\mathrm{TM}}$ to predict inflammation. The sensitivity, specificity, positive predictive value (PPV), negative predictive value, and accuracy were $96.5 \%, 50 \%, 66.6 \%$, $93.3 \%$, and $73.6 \%$, respectively, and use of the EGD in the lower third of the esophagus once again was associated with the highest accuracy. Finally, the interobserver and intraobserver agreement were calculated using a Kappa value for interobserver agreement of $0.85 \pm 0.13(0.59-1.11)$ and intraobserver agreement of $0.90 \pm 0.13(0.64-1.16)$.

\section{Discussion}

Approximately $20 \%$ of patients with NERD showed no evidence of microscopic esophagitis on biopsy [19]. However, it is estimated that NERD accounts for up to $60 \%$ to $70 \%$ of patients with GERD. Importantly, standard endoscopic evaluation is an inaccurate test for diagnosing reflux disease in patients with NERD, due to absence of visible lesions on endoscopy, making 
- Table 4 Contingency table comparing the esophagogastroduodenoscopy results with those of 24-hour pH-impedance monitoring tests and histopathological targeted biopsies.

\begin{tabular}{|c|c|c|c|c|c|}
\hline & Sensitivity \% (95\% IC) & Specificity \% (95\% IC) & PPV \% (95\% IC) & NPV \% (95\% IC) & Accuracy \% \\
\hline \multicolumn{6}{|c|}{24 hours pH-impedance monitoring test } \\
\hline EGD overall & $94.4(81.3-99.3)$ & $61.9(38.4-81.9)$ & $80.9(65.9-91.4)$ & $86.7(59.5-98.3)$ & 82.5 \\
\hline EGD lower third of esophagus & $88.9(73.9-96.9)$ & $85.7(63.7-96.9)$ & $91.4(76.9-98.2)$ & $81.8(59.7-94.8)$ & 87.7 \\
\hline EGD middle third of esophagus & $75.0(57.8-87.9)$ & $71.0(48.0-89.0)$ & $82.0(65.0-93.0)$ & $62.5(40.6-81.2)$ & 73.7 \\
\hline EGD upper third of esophagus & $80.6(63.9-91.8)$ & $66.7(43.0-85.4)$ & $80.6(63.9-91.8)$ & $66.7(43.0-85.4)$ & 75.4 \\
\hline \multicolumn{6}{|l|}{ Biopsy } \\
\hline EGD overall & $96.5(82.2-99.9)$ & $50.0(30.7-69.3)$ & $66.7(50.5-80.4)$ & $93.3(68.1-99.8)$ & 73.7 \\
\hline EGD lower third of esophagus & $92.3(74.9-99.1)$ & $64.5(45.3-80.8)$ & $68.6(50.7-83.2)$ & $90.9(70.8-98.9)$ & 77.2 \\
\hline EGD middle third of esophagus & $81.8(59.7-94.8)$ & $57.1(39.4-73.7)$ & $54.6(36.4-71.9)$ & $83.3(62.6-95.3)$ & 66.7 \\
\hline EGD upper third of esophagus & $89.5(66.9-98.7)$ & $50.0(33.4-66.6)$ & $47.2(30.4-64.5)$ & $90.5(69.6-98.8)$ & 63.1 \\
\hline
\end{tabular}

it necessary to further evaluate such patients with other diagnostic tests, such as $\mathrm{pH}$-impedance monitoring $[20,21]$.

Previous studies using different endoscopic technologies, such as high-definition and Lugol chromoendoscopy, attempted to identify minimal esophageal mucosal changes, such as erythema or the invisibility of vessels, in NERD. However, these lesions were found in only $43 \%$ of patients with low sensitivity and low interobserver agreement $[22,23]$.

Chromoendoscopy with or without optical magnification has been previously used to identify subtle esophageal changes and diagnose NERD [24-26]. Alterations such as punctuate erythema located above the Z-line are typically classified as caused by NERD, but the sensitivity for their detection is low (64\%), and the interobserver consistency displays poor agreement [25]. I-scan is useful for detecting Barrett's esophagus and reflux esophagitis [7]. Netinatsunton et al used I-scan to detect minimal change esophagitis (minute erosion, punctuate erythema and elongated pit pattern of gastric mucosa with triangular lesions); however, low sensitivity and PPV were described [8].

Digital chromoendoscopy with high-definition scopes using NBI has shown greater sensitivity than WLE for detecting inflammation in patients with suspected NERD. However, histopathological findings are more prevalent than mucosal changes detected using this imaging technique, such that use of biopsies is still necessary [16].

Recently, Sharma et al. used optical magnification with digital chromoendoscopy (NBI) to evaluate 80 patients with GERD. They found that these patients had increased vascularity at the squamocolumnar junction compared with control subjects. Although interobserver agreement for NBI was very good (increase number of IPCLs [ $\mathrm{K}=0.80]$, dilation [ $\mathrm{k}=0.75]$, and tortuosity $[k=0.89])$, intra-observer agreement was only modest (increase number of IPCLs [ $K=0.51]$, dilation [ $K=0.51]$, and tortuosity [ $k=0.39]$ [ [9]. However, this study has the limitation of defining GERD based on results of two validated questionnaires instead of using a 24-hour $\mathrm{pH}$-impedance monitoring test to confirm reflux. Moreover, the study was not originally designed to evaluate IPCLs in patients with NERD. Thus, an increase in acid exposure time was not confirmed in the subgroup of patients with NERD, and inflammation was not confirmed histologically.

The current study aimed to clarify whether digital chromoendoscopy using the OE system and optical magnification using Magniview scopes could be used to determine whether IPCLs can serve as an early sign of NERD. Therefore, we prospectively evaluated specific patients with NERD after excluding minimal change esophagitis via I-scan and confirming reflux using the 24-hour $\mathrm{pH}$-impedance monitoring test with additional histological evaluation. We found that IPCLs were observed in $94.4 \%$ of the NERD group, and the lower third segment of the esophagus was the most affected by these abnormalities $(P<0.05)$. By contrast, only $38 \%$ of control group patients had IPCLs. In addition, we found that increased numbers of IPCLs and IPCL dilation were the most commonly observed abnormalities, affecting $86.1 \%$ and $91.6 \%$ of patients in the NERD group, respectively. In addition, we demonstrated that IPCLs in patients with NERD are early microvascular inflammatory lesions in the lamina propria, which can be considered as early signs of NERD.

The current study has the advantage of a well-founded methodological design, in which patients with GERD and minimal change esophagitis were excluded using I-scan technology; more accurate gold standards for defining reflux and inflammation (24-hour $\mathrm{pH}$-impedance monitoring test and histological analysis) instead of the validated questionnaires used in previous studies were applied $[8,9]$. The study was specifically designed to evaluate IPCL characteristics in patients with NERD; thus, it allowed us to confirm that IPCL dilation and increases in numbers are abnormalities found in patients with NERD that might be considered as a diagnostic method.

The study did have some limitations. It was a non-randomized, single-center and single-blind study. Only patients with 
typical reflux disease (heartburn and/or regurgitation) were included. The generalizability of these findings needs to be evaluated, considering that MagniView scopes are not available in all endoscopic units. In addition, a practical limitation of optical magnification is the necessity of focusing the scope tip closer to the mucosa. Optical enhancement plus optical magnification for evaluating IPCLs as a diagnostic marker of NERD requires a learning curve to adjust scope maneuverability. In addition, longer time during endoscopy must be noted as a limitation.

\section{Conclusion}

In conclusion, use of the $\mathrm{OE}$ systemalong with Magniview scopes proved to be a useful method for detecting MMEL and predicting reflux in NERD patients. These technologies had high sensitivity, accuracy, and good interobserver and intraobserver agreement. In addition, presence of IPCLs was highly correlated with a positive diagnosis using 24-hour $\mathrm{pH}$-impedance monitoring test and histological inflammation, thus suggesting that other methods may not be essential to make a NERD diagnosis.

\section{Competing interests}

Dr. Robles-Medranda is a key opinion leader and consultant for Pentax Medical.

\section{References}

[1] Gomes CA Jr, Loução TS, Carpi G et al. A study on the diagnosis of minimal endoscopic lesions in nonerosive reflux esophagitis using computed virtual chromoendoscopy (FICE). Arq Gastroenterol 2011; 48: $167-170$

[2] Alzubaidi M, Gabbard S. GERD: Diagnosing and treating the burn. Cleve Clin J Med 2015; 82: 685-692

[3] Takeshima F, Hashiguchi K, Onitsuka Y et al. Clinical characteristics of patients with gastroesophageal reflux disease refractory to proton pump inhibitors and the effects of switching to $20 \mathrm{mg}$ esomeprazole on reflux symptoms and quality of life. Med Sci Monit 2015; 21: $4111-4121$

[4] Vela MF. Diagnostic work-up of GERD. Gastrointest Endoscopy Clin N Am 2014; 24: 655-666

[5] Dent J, Armstrong D, Delaney B et al. Symptom evaluation in reflux disease: workshop background, processes, terminology, recommendations, and discussion outputs. Gut 2004; 53: 1-24

[6] Kim MS, Choi SR, Roh MH et al. Efficacy of I-scan endoscopy in the diagnosis of gastroesophageal reflux disease with minimal change. Clin Endosc 2011; 44: 27-32

[7] Robles-Medranda C, Del Valle RS, Soria Alcívar M et al. Pentax I-SCAN with magnification for the identification of under diagnosis organic esophageal lesions (barret's esophagus and esophagitis) in patients with functional dyspepsia: a prospective study. Gastroenterology 2014; 146: S- 524
[8] Netinatsunton N, Sottisuporn J, Attasaranya S et al. i-Scan detection of minimal change esophagitis in dyspeptic patients with or without gastroesophageal reflux disease. BMC Gastroenterol 2016; 16: 1-8

[9] Sharma P, Wani S, Bansal S et al. A feasibility trial of narrow band imaging endoscopy in patients with gastroesophageal reflux disease. Gastroenterology 2007; 133: $454-464$

[10] Zavala-Gonzáles MA, Azamar-Jacome AA et al. Validation and diagnostic usefulness of gastroesophageal reflux disease questionnaire in a primary care level in Mexico. J Neurogastroenterol Motil 2014; 20: $475-482$

[11] Kodashima S, Fujishiro M. Novel image-enhaced endoscopy with iscan technology. World J Gastroenterol 2010; 16: 1043 -1049

[12] Lundell LR, Dent J, Bennett JR et al. Endoscopic assessment of esophagitis: clinical and functional correlates and further validation of the Los Angeles classification. Gut 1999; 45: $172-180$

[13] Kandulski A, Weigt J, Caro C et al. Esophageal intraluminal baseline impedance differentiates gastroesophageal reflux disease from functional heartburn. Clin Gastroenterol Hepatol 2015; 13: 1075-1081

[14] Kasap E, Zeybel M, Aşık G et al. Correlation among standard endoscopy, narrow band imaging, and histopathological findings in the diagnosis of nonerosive reflux disease. J Gastrointestin Liver Dis 2011; 20: $127-130$

[15] Lv J, Liu D, Ma SY et al. Investigation of relationships among gastroesophageal reflux disease subtypes using narrow band imaging magnifying endoscopy. World J Gastroenterol 2013; 19: 8391 -8397

[16] Zentilin P, Savarino V, Mastracci L et al. Reassessment of the diagnostic value of histology in patients with GERD, using multiple biopsy sites and an appropriate control group. Am J Gastroenterol 2005; 100: $2299-2306$

[17] Miyasaka M, Hirakawa M, Nakamura K et al. The endoscopic diagnosis of nonerosive reflux disease using flexible spectral imaging color enhancement image: feasibility trial. Dis Esophagus 2011; 24: 395-400

[18] Nandurkar S, Talley NJ, Martin C] et al. Esophageal histology does not provide additional useful information overclinical assessment in identifying reflux patients presenting for esophagogastroduodenoscopy. Dig Dis Sci 2000; 45: $217-224$

[19] Landis JR, Koch GG. The measurement of observer agreement for categorical data. Biometrics 1977; 33: 159-174

[20] Azumi T, Adachi K, Furuta K et al. Esophageal epithelial surface in patients with gastroesophageal reflux disease: An electron microscopic study. World J Gastroenterol 2008; 14: 5712-5716

[21] Gatopoulou A, Mimidis K, Giatromanolaki A et al. Impact of hiatal hernia on histological pattern of non-erosive reflux disease. BMC Gastroenterol 2005; 5: 2

[22] Falk GW. Is conventional endoscopic identification of non-erosive reflux disease adequate? Digestion 2008; 78: 17-23

[23] Gossner L. Potential contribution of novel imaging modalities in nonerosive reflux disease. Best Pract Res Clin Gastroenterol 2008; 22: $617-624$

[24] Kiesslich R, Kanzler S, Vieth M et al. Minimal chance esophagitis: prospective comparison of endoscopic and histological markers between patients with non-erosive reflux disease and normal controls using magnifying endoscopy. Dig Dis 2004; 22: 221-227

[25] Yoshikawa I, Yamasaki M, Yamasaki T et al. Lugol chromoendoscopy as a diagnostic tool in so-called endoscopy-negative GERD. Gastrointest Endosc 2005; 62: $698-703$

[26] Dy FT, Sollano J, Quino A et al. Lugol's chromoendoscopy improves the detection of esophagitis in patients with nonerosive reflux disease. Gastrointest Endosc 2004; 59: 243 